\title{
Association of the NOTCH4 Gene Polymorphism rs204993 with Schizophrenia in the Chinese Han Population
}

\author{
Bao Zhang, ${ }^{1}$ Qian Rui Fan, ${ }^{1}$ Wen Hao Li, ${ }^{1}$ Ning Lu, ${ }^{2}$ Dong Ke Fu, ${ }^{1}$ Yan Jie Kang, \\ Na Wang, ${ }^{1}$ Teng Li, ${ }^{1}$ Xiao Peng Wen, ${ }^{3}$ and Da Xu Li ${ }^{4}$ \\ ${ }^{1}$ College of Medicine \& Forensic, Health Science Center, Xian Jiaotong University, Xian 710061, China \\ ${ }^{2}$ Department of Food and Biological Engineering, Henan Industry \& Trade Vocational College, Henan, Zhengzhou 451191, China \\ ${ }^{3}$ Department of Thoracic Surgery, The First Affiliated Hospital, Xian Jiaotong University, Xian 710061, China \\ ${ }^{4}$ Department of Stomatology, The First Affiliated Hospital, Xian Jiaotong University, Xian 710061, China
}

Correspondence should be addressed to Xiao Peng Wen; tp1005@163.com and Da Xu Li; sheraer@gmail.com

Received 14 July 2015; Accepted 17 September 2015

Academic Editor: Eberval G. Figueiredo

Copyright (C) 2015 Bao Zhang et al. This is an open access article distributed under the Creative Commons Attribution License, which permits unrestricted use, distribution, and reproduction in any medium, provided the original work is properly cited.

\begin{abstract}
NOTCH4 regulates signaling pathways associated with neuronal maturation, a process involved in the development and patterning of the central nervous system. The NOTCH4 gene has also been identified as a possible susceptibility gene for schizophrenia (SCZ). The objective of this study was to examine the relationship between NOTCH4 polymorphisms and SCZ in the Chinese Han population. The rs2071287 and rs204993 polymorphisms of the NOTCH4 gene were analyzed in 443 patients with SCZ and 628 controls of Han Chinese descent. Single SNP allele-, genotype-, and gender-specific associations were analyzed using different models (i.e., additive, dominant, and recessive models). This association study revealed that the rs204993 polymorphism is significantly associated with susceptibility for SCZ and that the AA genotype of rs204993 is associated with a higher risk for SCZ $(P=0.027 ; \mathrm{OR}=1.460 ; 95 \% \mathrm{CI}, 1.043-2.054)$. Our data are consistent with those obtained in previous studies that suggested that rs204993 is associated with SCZ and that the AA genotype of rs204993 demonstrates a higher risk. Further large-scale association analyses in Han Chinese populations are warranted.
\end{abstract}

\section{Introduction}

Schizophrenia (SCZ) is a chronic, severe psychotic mental disorder characterized by both positive (e.g., delusions, hallucinations, and thought disorders) and negative symptoms (e.g., social withdrawal, apathy, and cognitive impairment) [1]. As a serious neurological disability, SCZ affects approximately $1 \%$ of the general population worldwide and is regarded as a major public health problem, ranking ninth in terms of global disease burden [2]. Based on evidence from family-based, twin and adoption studies, which have implicated numerous genes in the etiology of SCZ, the disorder is currently understood as a polygenic neurodevelopment disorder caused by the interplay between environmental factors and genetics [3]. Although genome-wide association studies (GWAS) and candidate gene approaches to SCZ have produced many positive results $[4,5]$, only a small proportion of the genes have been consistently studied across multiple different populations. Therefore, the exploration of the true etiology and genetic mechanisms underlying the associations between genes and SCZ in various genetically independent populations is necessary.

The NOTCH4 (neurogenic locus notch homolog protein 4) gene, located at the centromeric end of the HLA class III region, has been implicated in SCZ based on evidence from several genetic studies [6]. Some evidence has suggested that NOTCH4 polymorphisms, including microsatellite and SNPs (e.g., rs3132935, rs3131296, and rs3809842), have been described [7-10]. More recently, a GWAS study identified rs2071287 of the NOTCH4 gene as the most statistically significant marker, which was confirmed through a followup analysis in a Japanese population [11]. Furthermore, rs2071287 and rs3131296, which display very strong LD with each other, were identified as the SNPs that are most likely to 
be functionally relevant to SCZ etiology, suggesting that it is necessary to determine the association of rs2071287 with SCZ $[12,13]$. Another SNP, rs204993, was found to be significantly associated with SCZ through a family-based association study of 218 Taiwan nuclear families [14]. To date, few studies have investigated the association of NOTCH4 with SCZ in the Chinese population, particularly regarding the SNPs rs2071287 and rs204993. Moreover, a genetic model describing the importance of the NOTCH4 gene in SCZ has not yet been elucidated.

Therefore, we performed a case-control study to examine the possibility of NOTCH4 polymorphisms being associated with SCZ in the Chinese Han population and to determine the inheritance models for the association study.

\section{Materials and Methods}

2.1. Subjects. The case-control samples included $443 \mathrm{SCZ}$ cases (224 females and 219 males, mean age: $36.1 \pm 10.2$ years) and 628 healthy controls (358 females and 270 males, mean age: $35.7 \pm 9.7$ years). The subjects were all original northern Han Chinese recruited from The First Affiliated Hospital of Xi'an Jiaotong University. The available information (i.e., personal history, hospital record, and family-history report) was analyzed based on The Fourth Edition of the Diagnostic and Statistical Manual of Mental Disorders (DSM-IV) to obtain a consensus diagnosis agreed on by at least two experienced senior psychiatrists. After undergoing physical examinations, subjects with substance-induced psychotic disorders, learning disabilities, head injuries, and other symptomatic psychoses were excluded from the present study. Moreover, the controls were confirmed to not have any mental illness and were matched with the patients in age, sex, and educational level. Informed consent was obtained from all of the participants. The study was approved by the Xi'an Jiaotong University School of Medicine and The First Affiliated Hospital of Xian Jiaotong University.

2.2. SNP Genotyping. Two SNPs, rs2071287 and rs204993, showed significant associations with SCZ in previous manuscripts $[11,14]$. They were located in the intron and $3^{\prime}$ untranslated region of the NOTCH4 gene and detected in the present study. Genomic DNA was extracted from the peripheral blood leukocytes of all of the subjects based on the manufacturer's recommendations (Omega, Bio-tek, GA, USA). All of the SNPs were genotyped by the SNPscan technique (Genesky Biotechnologies Inc., Shanghai, China) according to a previous study [15]. Five percent of the samples with high DNA quality were randomly repeated to guarantee the genotyping quality. The average genotype call rate for all of the markers was $96.5 \%$.

2.3. Statistical Analysis. The $\mathrm{G}^{*}$ Power program was used to calculate the statistical power of our sample size according to Cohen's method [16]. The sample size exhibited $>80 \%$ power for the detection of significant $(P<0.05)$ associations at an effect size index of 0.1 (corresponding to a "weak" gene effect).

Hardy-Weinberg equilibrium (HWE) was detected for the SNP genotypes by the Chi-square test, which was also used to calculate the differences in the genotype and allele frequencies between the cases and controls. We performed logistic regression analyses to identify SCZ-associated SNPs by their odds ratios (OR), 95\% confidence intervals (CIs), and corresponding $P$ values. Furthermore, single SNP analyses were performed using multiple inheritance models, namely, additive, dominant, and recessive models, similarly to an earlier study [15]. Moreover, stratified analyses were conducted to detect whether differences in gender and genetics influenced such associations. All of the statistical analyses were conducted using PLINK (version 1.07). Differences were considered significant when the $P$ value was 0.05 .

\section{Results}

For the study, 443 eligible individuals with schizophrenia and 628 healthy controls were enrolled. Two SNPs (rs2071287 and rs204993) in the NOCTH4 gene were genotyped and analysed.

3.1. Hardy-Weinberg Equilibria of Two Variants. None of the rs2071287 or rs204993 SNPs showed deviation from HardyWeinberg equilibria in the control population $(P=0.18$, and $P=0.79$, resp.).

3.2. Association with the Two Variants. The allele and genotype frequencies of the two SNPs (rs2071287 and rs204993) are shown in Table 1 . The estimated risks associated with these polymorphisms in the SCZ patients and the healthy controls were tested according to three models (i.e., dominant, recessive, and addictive models), also illustrated in Table 1. Considering all the subjects, the allele frequencies of rs2071287 and rs204993 for the patients with SCZ were not significantly different from those of the control group. However, notably, genotype association analysis for rs204993 suggested a significant association with SCZ under a recessive model, and the AA genotype of rs204993 was associated with a higher risk of SCZ $(P=0.027$; OR $=1.460 ; 95 \% \mathrm{CI}, 1.043-2.054)$.

\subsection{Association with the Two Variants of the Gender-Specific} Analyses. To examine whether gender would play a role in the association, we analyzed our data by separating males and females on the basis of results above (Table 2). Furthermore, we found that rs204993 showed marginally significant genotypic $(P=0.084 ; \mathrm{OR}=1.561 ; 95 \% \mathrm{CI}, 0.939-2.596)$ associations with SCZ in males, but not in females.

\section{Discussion}

The NOTCH4 gene located on 6p21.3 encodes a member of the NOTCH family, the members of which play a pivotal role in deciding cell fate, particularly during the neurodevelopmental process to promote proliferative signaling [14, 17-19]. In particular, NOTCH4 regulates the signaling of the maturation of neurons and glia from neural stem cells [13, 20, 21]. As an important neurodevelopment-related gene, transcripts of NOTCH4 can be detected in the developing nervous system [22], which makes NOTCH4 a potential candidate gene for neurodevelopment disorders, such as SCZ [23]. Previous 
TABLE 1: Analysis of the allele and genotype frequencies of a single SNP association.

\begin{tabular}{|c|c|c|c|c|c|c|c|c|c|c|c|}
\hline \multirow{2}{*}{$\begin{array}{l}\text { Makers } \\
\text { Rs204993 }\end{array}$} & \multicolumn{2}{|c|}{ Allele freq. (\%) } & \multirow[t]{2}{*}{$P$ value $^{\mathrm{a}}$} & \multirow[t]{2}{*}{$\begin{array}{c}\text { OR } \\
(95 \% \mathrm{CI}) \\
\end{array}$} & \multicolumn{3}{|c|}{ Genotype $(N)$} & \multirow[t]{2}{*}{$\begin{array}{c}\text { HWE } \\
P \text { value }\end{array}$} & \multirow[t]{2}{*}{ Model } & \multirow[t]{2}{*}{$\begin{array}{c}\text { OR } \\
(95 \% \mathrm{CI}) \\
\end{array}$} & \multirow[t]{2}{*}{$P$ value } \\
\hline & G & $\mathrm{A}$ & & & GG & AG & AA & & & & \\
\hline SCZ & 41.3 & 58.7 & 0.223 & $\begin{array}{c}1.115 \\
(0.936-1.329)\end{array}$ & 78 & 210 & 155 & 0.182 & Add & $\begin{array}{c}1.121 \\
(0.937-1.342)\end{array}$ & 0.213 \\
\hline \multirow[t]{2}{*}{ CTR } & 38.7 & 61.3 & & & 80 & 326 & 222 & & Dom & $\begin{array}{c}1.016 \\
(0.788-1.311)\end{array}$ & 0.903 \\
\hline & & & & & & & & & $\operatorname{Rec}$ & $\begin{array}{c}1.464 \\
(1.043-2.054)\end{array}$ & 0.027 \\
\hline Rs2071287 & A & G & & & $\mathrm{AA}$ & AG & GG & & & & \\
\hline SCZ & 38.0 & 62.0 & 0.530 & $\begin{array}{c}1.059 \\
(0.886-1.264)\end{array}$ & 68 & 201 & 174 & 0.794 & Add & $\begin{array}{c}1.058 \\
(0.887-1.263)\end{array}$ & 0.532 \\
\hline \multirow[t]{2}{*}{ CTR } & 36.7 & 63.3 & & & 83 & 295 & 250 & & Dom & $\begin{array}{c}1.022 \\
(0.797-1.311)\end{array}$ & 0.861 \\
\hline & & & & & & & & & $\operatorname{Rec}$ & $\begin{array}{c}1.191 \\
(0.842-1.682)\end{array}$ & 0.324 \\
\hline
\end{tabular}

SCZ: schizophrenia; CTR: control; CI: confidence interval; OR: odds ratio.

Significant $P$ values and HWE $P$ values are shown in italic bold font.

${ }^{\text {a }} P$ values of the normal Chi-square statistics.

Add: addictive model; Dom: dominant model; Rec: recessive model.

TABLE 2: Analysis of sex-specific allele and genotype associations.

\begin{tabular}{|c|c|c|c|c|c|c|c|c|c|c|c|}
\hline \multirow{2}{*}{\multicolumn{2}{|c|}{$\begin{array}{c}\text { Markers and sex } \\
\text { rs204993 }\end{array}$}} & \multicolumn{2}{|c|}{ Allele freq. (\%) } & \multirow{3}{*}{$\begin{array}{c}P \text { value }^{\mathrm{a}} \\
0.366\end{array}$} & \multirow{3}{*}{$\begin{array}{c}\text { OR } \\
(95 \% \mathrm{CI}) \\
\\
\\
1.118 \\
(0.878-1.422)\end{array}$} & \multicolumn{3}{|c|}{ Genotype $(N)$} & \multirow{3}{*}{$\begin{array}{c}\text { Model } \\
\text { Add }\end{array}$} & \multirow{3}{*}{$\begin{array}{c}\text { OR } \\
(95 \% \mathrm{CI}) \\
\\
1.120 \\
(0.878-1.429)\end{array}$} & \multirow{3}{*}{$\begin{array}{c}P \text { value }^{\mathrm{a}} \\
0.362\end{array}$} \\
\hline & & G & $\mathrm{A}$ & & & \multirow{2}{*}{$\begin{array}{l}\text { GG } \\
40\end{array}$} & \multirow{2}{*}{$\begin{array}{l}\mathrm{AG} \\
104\end{array}$} & \multirow{2}{*}{$\begin{array}{l}\text { AA } \\
80\end{array}$} & & & \\
\hline $\mathrm{F}$ & SCZ & 41.1 & 58.9 & & & & & & & & \\
\hline & CTR & 38.4 & 61.6 & & & 48 & 179 & 131 & Dom & $\begin{array}{c}1.039 \\
(0.734-1.471)\end{array}$ & 0.830 \\
\hline & & & & & & & & & $\operatorname{Rec}$ & $\begin{array}{c}3.242 \\
(0.589-17.85)\end{array}$ & 0.145 \\
\hline \multirow[t]{3}{*}{ M } & SCZ & 41.6 & 89.7 & 0.432 & $\begin{array}{c}1.109 \\
(0.857-1.433)\end{array}$ & 38 & 106 & 75 & Add & $\begin{array}{c}1.118 \\
(0.856-1.461)\end{array}$ & 0.414 \\
\hline & CTR & 39.1 & 89.6 & & & 32 & 147 & 91 & Dom & $\begin{array}{c}0.976 \\
(0.670-1.422)\end{array}$ & 0.899 \\
\hline & & & & & & & & & $\operatorname{Rec}$ & $\begin{array}{c}1.561 \\
(0.939-2.596)\end{array}$ & 0.084 \\
\hline \multicolumn{2}{|c|}{ rs2071287 } & $\mathrm{T}$ & $\mathrm{C}$ & & & $\mathrm{TT}$ & TC & $\mathrm{CC}$ & & & \\
\hline \multirow[t]{3}{*}{$\mathrm{F}$} & SCZ & 39.3 & 60.7 & 0.098 & $\begin{array}{c}1.229 \\
(0.962-1.569)\end{array}$ & 36 & 104 & 84 & Add & $\begin{array}{c}1.222 \\
(0.960-1.555)\end{array}$ & 0.104 \\
\hline & CTR & 34.5 & 65.5 & & & 45 & 157 & 156 & Dom & $\begin{array}{c}1.287 \\
(0.915-1.812)\end{array}$ & 0.148 \\
\hline & & & & & & & & & $\operatorname{Rec}$ & $\begin{array}{c}1.332 \\
(0.829-2.14)\end{array}$ & 0.236 \\
\hline \multirow[t]{3}{*}{ M } & SCZ & 36.8 & 63.2 & 0.358 & $\begin{array}{c}1.885 \\
(0.683-1.148)\end{array}$ & 32 & 97 & 90 & Add & $\begin{array}{c}0.884 \\
(0.680-1.148)\end{array}$ & 0.355 \\
\hline & CTR & 39.6 & 60.4 & & & 44 & 138 & 94 & Dom & $\begin{array}{c}0.766 \\
(0.530-1.106)\end{array}$ & 0.154 \\
\hline & & & & & & & & & $\operatorname{Rec}$ & $\begin{array}{c}1.045 \\
(0.629-1.737)\end{array}$ & 0.866 \\
\hline
\end{tabular}

SCZ: schizophrenia; CTR: control; CI: confidence interval; OR: odds ratio.

${ }^{\mathrm{a}} P$ values of the normal Chi-square statistics.

Add: addictive model; Dom: dominant model; Rec: recessive model. 
studies identified NOTCH4 polymorphisms implicated in the risk of SCZ. In this study, two SNPs of the NOTCH4 gene were detected to confirm their association with SCZ in the Han Chinese population. We found that the allele and genotype distributions of rs2071287 demonstrated insignificant associations. Notably, the genotype distribution of the rs204993 polymorphism demonstrated a significant association with SCZ under a recessive model $(P=0.027)$.

However, a previous GWAS study, as well as a follow-up analysis, revealed that rs2071287 was associated with SCZ in the Japanese population $[11,24]$. In contrast, our results are consistent with other studies that reported an insignificant association between the rs2071287 polymorphism and SCZ $[25,26]$. The rs2071287 frequency of the "A" allele differs considerably ( 0.31 for Japanese, 0.36 for Bulgarian, and 0.38 for Chinese Han), which suggests that subjects from different ethnic backgrounds may exhibit SCZ-related genetic heterogeneity. Hence, our findings suggest that this SNP may represent a risk for SCZ under certain ethnic backgrounds rather than universally.

A family-based association study preliminarily showed an association between rs204993 and SCZ. Consistent with this finding, the present case-control study suggested that NOTCH4 rs204993 is significantly associated with SCZ. The rs204993 polymorphism of the NOTCH4 gene is located in the $3^{\prime}$ UTR region. And rs204993 "A" allele is the major allele in the study population, suggesting this polymorphism may be in LD with another potentially functional SNP that affects NOTCH4 expression. Furthermore, rs204993 has also been mapped to the adjacent gene PBX2, which is also located within the SCZ-related region in the major histocompatibility complex (MHC) [27].

Based on the available findings, because family-based and case-control association studies have demonstrated significant associations between rs204993 and SCZ, we can only predict that rs204993 associates with SCZ as it is in LD with another functional SNP which may have an effect on gene expression. We hypothesize that further functional exploration (such as in vitro and in vivo experimentation) of this polymorphism could lead to an important understanding of the genetic pathophysiology of schizophrenia.

This study has several limitations that need to be addressed. First, the relatively small sample size of our experimental study limits the interpretation of the results for the Chinese Han population. However, the observation of a statistically significant association is encouraging. Furthermore, this study was performed at a single center, which may potentially limit the generalizability of the findings. Moreover, other SNPs in the candidate region/gene in addition to the reported risk-related SNP may account for the association with schizophrenia detected in different populations. Despite these limitations, our current study provides useful data for a future meta-analysis of these psychosis markers.

In summary, our data support an association between NOTCH4 and SCZ. However, further studies using larger numbers of samples of different ethnic groups should be performed to determine the validity of such relationships.

\section{Conflict of Interests}

The authors declare that they have no conflict of interests.

\section{Authors' Contribution}

Bao Zhang and Qian Rui Fan contributed equally to this work.

\section{Acknowledgments}

The authors are grateful to all of the participants who donated their time and samples for this study. In addition, the authors thank all of the donors for their assistance in accessing collections and their advice and comments during the preparation of this paper. This work was supported by the National Natural Science Foundation of China (Grant no. 31301949), the National Science Foundation for PostDoctoral Scientists of China (Grant no. 2013M532056), and the National Science Foundation for Post-Doctoral Scientists of Shaanxi.

\section{References}

[1] N. Andreasen, "Symptoms, signs, and diagnosis of schizophrenia," The Lancet, vol. 346, no. 8973, pp. 477-481, 1995.

[2] L. Halley, M. K. Doherty, I. L. Megson, N. McNamara, A. Gadja, and J. Wei, "Search for schizophrenia susceptibility variants at the HLA-DRB1 locus among a British population," Immunogenetics, vol. 65, no. 1, pp. 1-7, 2013.

[3] A. Yuan, Z. Yi, Q. Wang et al., "ANK3 as a risk gene for schizophrenia: new data in han Chinese and meta analysis," American Journal of Medical Genetics, Part B: Neuropsychiatric Genetics, vol. 159, no. 8, pp. 997-1005, 2012.

[4] X.-X. Yang, A.-N. Zhu, F.-X. Li, Z.-X. Zhang, and M. Li, "Neurogenic locus notch homolog protein 4 and brain-derived neurotrophic factor variants combined effect on schizophrenia susceptibility," Acta Neuropsychiatrica, vol. 25, no. 6, pp. 356-360, 2013.

[5] S. Ripke, A. R. Sanders, K. S. Kendler et al., "Genome-wide association study identifies five new schizophrenia loci," Nature Genetics, vol. 43, no. 10, pp. 969-976, 2011.

[6] J. Wei and G. P. Hemmings, "The NOTCH4 locus is associated with susceptibility to schizophrenia," Nature Genetics, vol. 25, no. 4, pp. 376-377, 2000.

[7] H. Ujike, Y. Takehisa, M. Takaki et al., "NOTCH4 gene polymorphism and susceptibility to schizophrenia and schizoaffective disorder," Neuroscience Letters, vol. 301, no. 1, pp. 41-44, 2001.

[8] S. Prathikanti, T. G. Schulze, Y.-S. Chen et al., "Neither singlemarker nor haplotype analyses support an association between genetic variation near NOTCH4 and bipolar disorder," American Journal of Medical Genetics Part B: Neuropsychiatric Genetics, vol. 131, no. 1, pp. 10-15, 2004.

[9] M. Tochigi, X. Zhang, T. Umekage et al., "Association of six polymorphisms of the NOTCH4 gene with schizophrenia in the Japanese population," American Journal of Medical Genetics: Neuropsychiatric Genetics, vol. 128, no. 1, pp. 37-40, 2004.

[10] A. D. Skol, K. A. Young, D. W. Tsuang et al., "Modest evidence for linkage and possible confirmation of association between 
NOTCH4 and schizophrenia in a large veterans affairs cooperative study sample," American Journal of Medical GeneticsNeuropsychiatric Genetics, vol. 118, no. 1, pp. 8-15, 2003.

[11] M. Ikeda, B. Aleksic, Y. Kinoshita et al., "Genome-wide association study of schizophrenia in a Japanese population," Biological Psychiatry, vol. 69, no. 5, pp. 472-478, 2011.

[12] N. C. Allen, S. Bagade, M. B. McQueen et al., "Systematic metaanalyses and field synopsis of genetic association studies in schizophrenia: the SzGene database," Nature Genetics, vol. 40, no. 7, pp. 827-834, 2008.

[13] C. Shayevitz, O. S. Cohen, S. V. Faraone, and S. J. Glatt, "A re-review of the association between the NOTCH4 locus and schizophrenia," American Journal of Medical Genetics B: Neuropsychiatric Genetics, vol. 159, no. 5, pp. 477-483, 2012.

[14] C.-M. Liu, Y.-L. Liu, C. S.-J. Fann et al., "Association evidence of schizophrenia with distal genomic region of NOTCH4 in Taiwanese families," Genes, Brain and Behavior, vol. 6, no. 6, pp. 497-502, 2007.

[15] B. Zhang, D. X. Li, N. Lu, Q. Fan, W. Li, and Z. Feng, "Lack of association between the TSPAN18 gene and schizophrenia based on new data from han Chinese and a meta-analysis," International Journal of Molecular Sciences, vol. 16, no. 6, pp. 11864-11872, 2015.

[16] F. Faul, E. Erdfelder, A.-G. Lang, and A. Buchner, "G* Power 3: a flexible statistical power analysis program for the social, behavioral, and biomedical sciences," Behavior Research Methods, vol. 39, no. 2, pp. 175-191, 2007.

[17] J. Huang, S. Yoshimura, N. Isobe et al., "A NOTCH4 missense mutation confers resistance to multiple sclerosis in Japanese," Multiple Sclerosis, vol. 19, no. 13, pp. 1696-1703, 2013.

[18] U.-M. Fiúza and A. M. Arias, "Cell and molecular biology of Notch," Journal of Endocrinology, vol. 194, no. 3, pp. 459-474, 2007.

[19] N. Šestan, S. Artavanis-Tsakonas, and P. Rakic, "Contactdependent inhibition of cortical neurite growth mediated by Notch signaling," Science, vol. 286, no. 5440, pp. 741-746, 1999.

[20] T. Terzić, M. Kastelic, V. Dolžan, and B. K. Plesničar, "Genetic variability testing of neurodevelopmental genes in schizophrenic patients," Journal of Molecular Neuroscience, vol. 56, no. 1, pp. 205-211, 2015.

[21] L. Grandbarbe, J. Bouissac, M. Rand, M. Hrabé de Angelis, S. Artavanis-Tsakonas, and E. Mohier, "Delta-Notch signaling controls the generation of neurons/glia from neural stem cells in a stepwise process," Development, vol. 130, no. 7, pp. 1391-1402, 2003.

[22] H. Uyttendaele, G. Marazzi, G. Wu, Q. Yan, D. Sassoon, and J. Kitajewski, "Notch4/int-3, a mammary proto-oncogene, is an endothelial cell-specific mammalian Notch gene," Development, vol. 122, no. 7, pp. 2251-2259, 1996.

[23] D. A. Lewis and P. Levitt, "Schizophrenia as a disorder of neurodevelopment," Annual Review of Neuroscience, vol. 25, pp. 409-432, 2002.

[24] M. Ikeda, B. Aleksic, K. Yamada et al., "Genetic evidence for association between NOTCH4 and schizophrenia supported by a GWAS follow-up study in a Japanese population," Molecular Psychiatry, vol. 18, no. 6, pp. 636-638, 2013.

[25] E. T. Betcheva, T. Mushiroda, A. Takahashi et al., "Case-control association study of 59 candidate genes reveals the DRD2 SNP rs6277 (C957T) as the only susceptibility factor for schizophrenia in the Bulgarian population," Journal of Human Genetics, vol. 54, no. 2, pp. 98-107, 2009.
[26] N. Shibata, T. Ohnuma, S. Higashi et al., "Genetic association between Notch4 polymorphisms and Japanese schizophrenics," Psychiatric Genetics, vol. 16, no. 2, pp. 77-79, 2006.

[27] B. Aguado and R. D. Campbell, "The novel gene g17, located in the human major histocompatibility complex, encodes PBX2, a homeodomain-containing protein," Genomics, vol. 25, no. 3, pp. 650-659, 1995. 


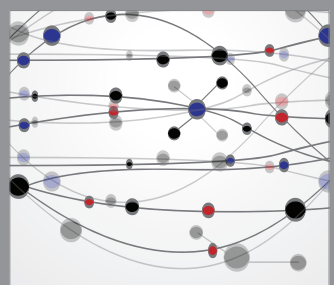

The Scientific World Journal
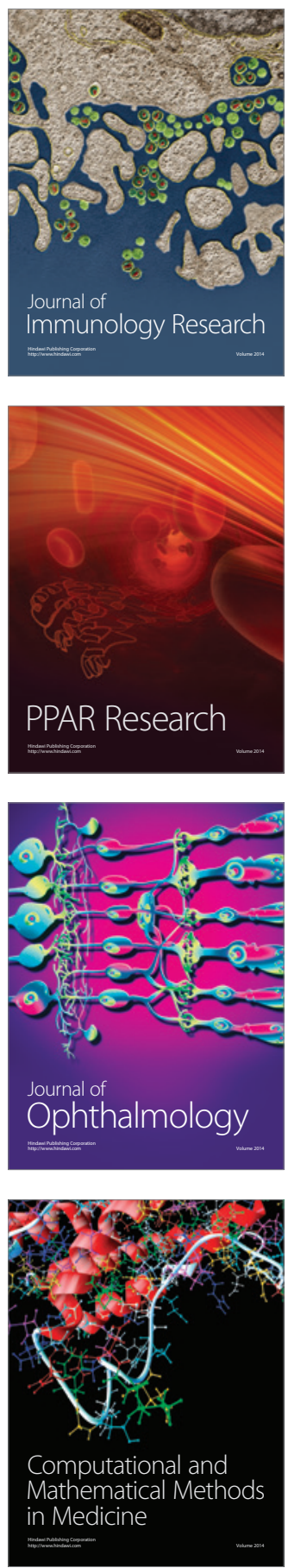

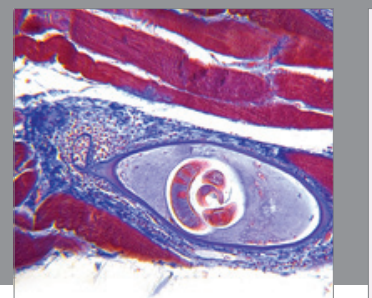

Gastroenterology

Research and Practice
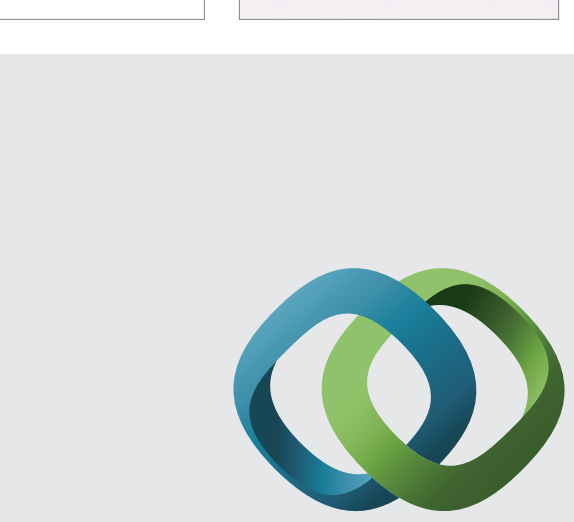

\section{Hindawi}

Submit your manuscripts at

http://www.hindawi.com
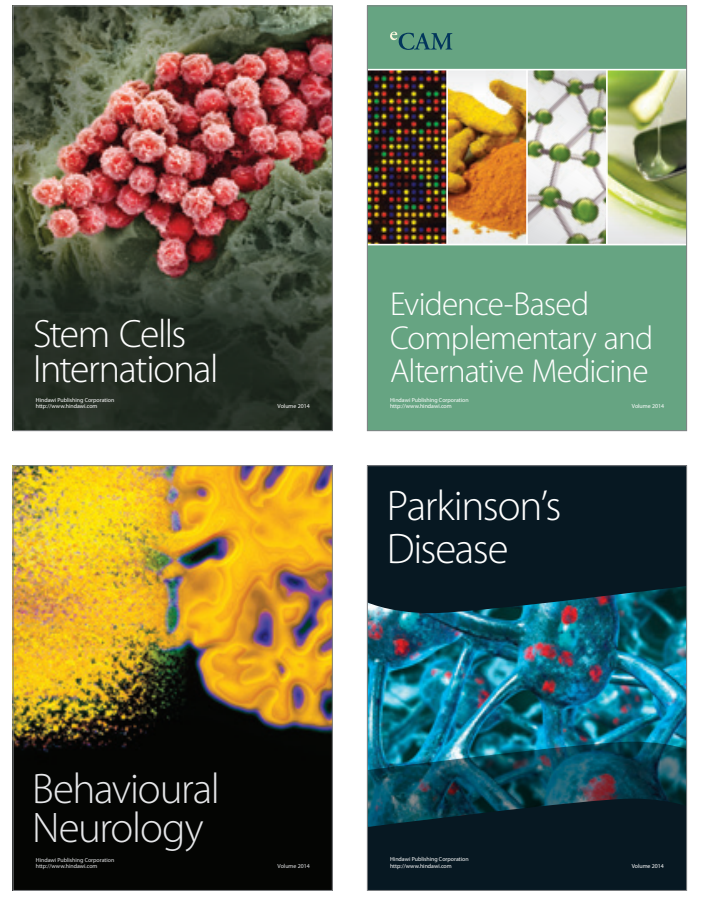
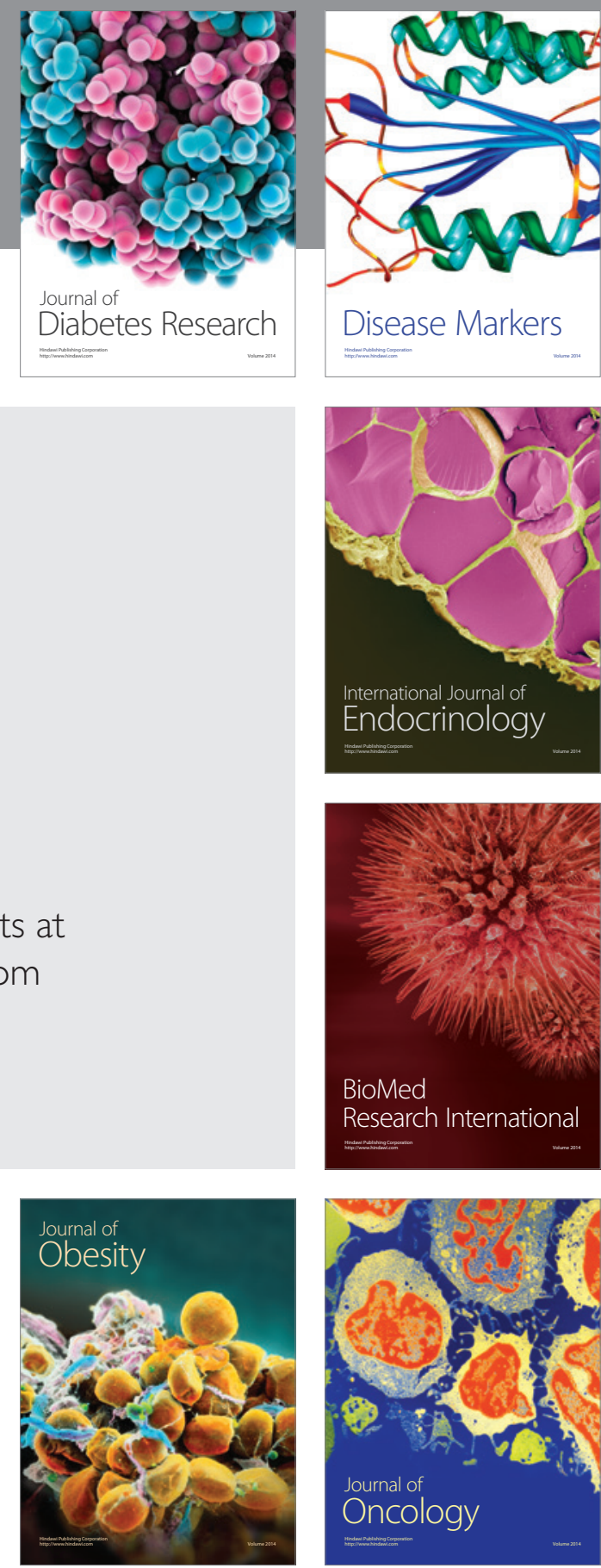

Disease Markers
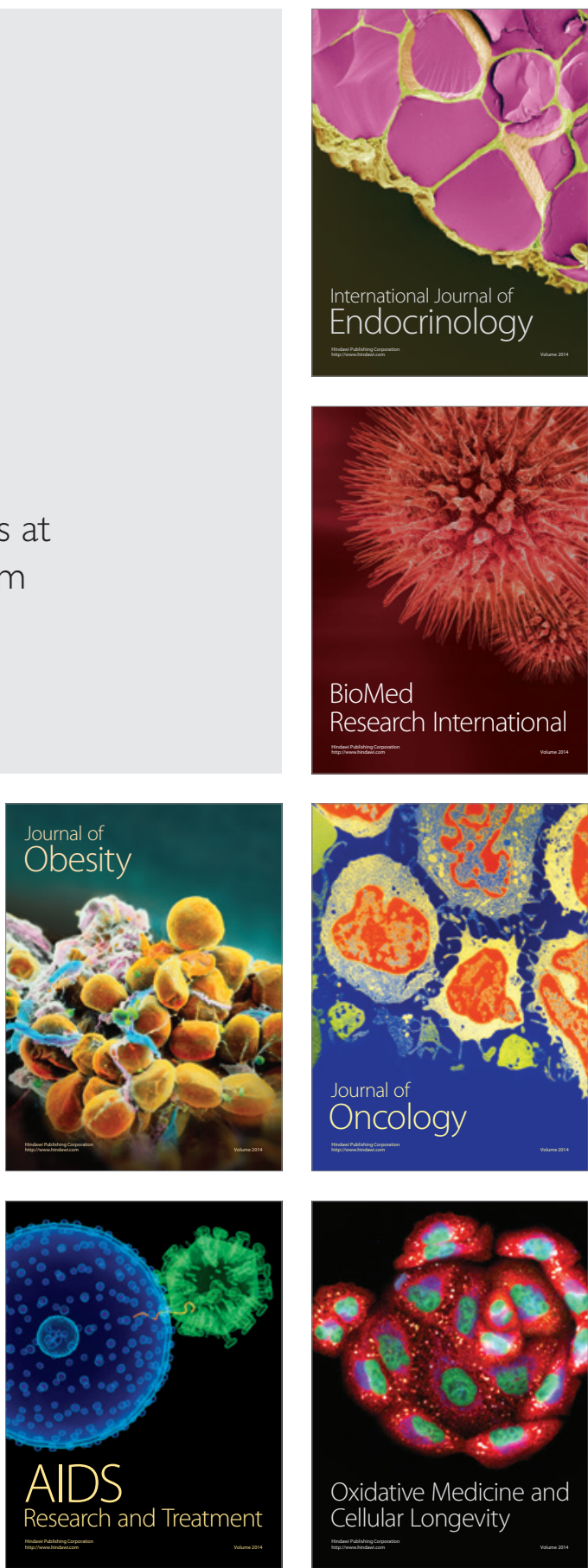